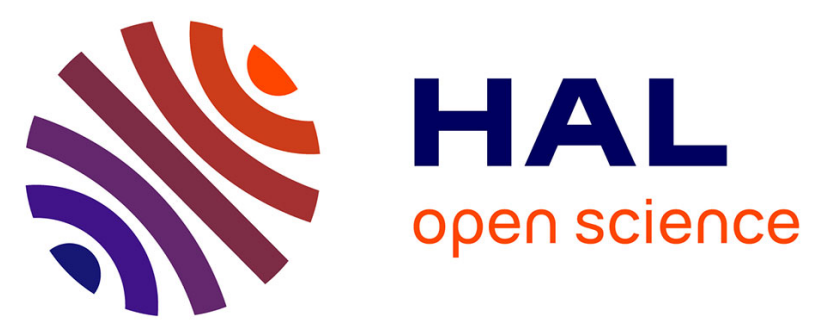

\title{
Novel approach for nano-patterning magnetic tunnel junctions stacks at narrow pitch: A route towards high density STT-MRAM applications
}

V. D. Nguyen, P. Sabon, J. Chatterjee, L. Tillie, P. Veloso Coelho, S. Auffret, R. Sousa, L. Prejbeanu, E. Gautier, L. Vila, et al.

\section{To cite this version:}

V. D. Nguyen, P. Sabon, J. Chatterjee, L. Tillie, P. Veloso Coelho, et al.. Novel approach for nanopatterning magnetic tunnel junctions stacks at narrow pitch: A route towards high density STTMRAM applications. 2017 IEEE International Electron Devices Meeting (IEDM), Dec 2017, San Francisco, CA, United States. 10.1109/IEDM.2017.8268517 . hal-01763666

\section{HAL Id: hal-01763666 https://hal.science/hal-01763666}

Submitted on 26 Jun 2018

HAL is a multi-disciplinary open access archive for the deposit and dissemination of scientific research documents, whether they are published or not. The documents may come from teaching and research institutions in France or abroad, or from public or private research centers.
L'archive ouverte pluridisciplinaire HAL, est destinée au dépôt et à la diffusion de documents scientifiques de niveau recherche, publiés ou non, émanant des établissements d'enseignement et de recherche français ou étrangers, des laboratoires publics ou privés. 


\title{
Novel approach for nano-patterning magnetic tunnel junctions stacks at narrow pitch: A route towards high density STT-MRAM applications
}

\author{
V. D. Nguyen ${ }^{1,2,3}$, P. Sabon ${ }^{1,2,3}$, J. Chatterjee ${ }^{1,2,3}$, L. Tille ${ }^{1,2,3}$, P. Veloso Coelho ${ }^{1,2,3}$, S. Auffret ${ }^{1,2,3}$, \\ R. Sousa ${ }^{1,2,3}$, L. Prejbeanu ${ }^{1,2,3}$, E. Gautier ${ }^{1,2,3}$, L. Vila ${ }^{1,2,3}$ and B. Dieny ${ }^{1,2,3}$ \\ ${ }^{1}$ Grenoble Alpes Univ., F-38000 Grenoble \\ ${ }^{2}$ CEA, INAC-SPINTEC, F-38000 Grenoble \\ ${ }^{3}$ CNRS, SPINTEC, F-38000 Grenoble \\ Email: vandai.nguyen@cea.fr
}

\begin{abstract}
Nano-patterning magnetic tunnel junction (MTJ) cells at low dimension and very dense pitch remains a challenge for high density STT-MRAM due to the difficulty of MTJ stacks etching. To circumvent this etching issue, this paper demonstrates a novel scalable approach for MTJ nanopatterning at very narrow pitch (pitch $=1.5 \mathrm{~F}, \mathrm{~F}=\mathrm{MTJ}$ dot diameter) by growing the MTJ material on pre-patterned conducting non-magnetic pillars without post-deposition etching. Advantageously, these pillars could be the vias connecting the different metal levels in CMOS technology. Structural, magnetic and transport properties of so prepared $\mathrm{MgO}$-based MTJs were investigated. The comparison with those obtained by conventional ion beam etching (IBE) shows that this novel approach is quite promising to circumvent the issue of MTJ etching for high density MRAM.
\end{abstract}

\section{INTRODUCTION}

Spin-Transfer-Torque Magnetic Random Access Memory (STT-MRAM) based on out-of-plane magnetized MTJ (pMTJ) is one of the most promising emerging non-volatile memory technologies. Indeed, it combines a unique set of assets: quasiinfinite write endurance, high speed, low power consumption and scalability. Embedded STT-MRAM are about to enter in volume production for e-FLASH replacement. For this type of applications not requiring very high memory density, the preferred etching technique is still IBE [1]. However this technique is not appropriate for high density memory due to shadowing effects (see Fig.1). This effect worsens as the memory pitch shrinks below typically $5 \mathrm{~F}$ resulting in a poor control of the critical dimension at very dense pitch. Besides it is difficult to implement on large wafer with good uniformity [2]. Reactive ion etching (RIE) was also tried for MTJs patterning with various gas but was found to be very complex due to the heterogeneous nature of the MTJ stacks and to cause corrosion and delamination of the magnetic materials [3]. Therefore, to be able to use STT-MRAM as a dense working memory, a new method for nanopatterning MTJ elements at small feature size $(\mathrm{F}<20 \mathrm{~nm})$ and narrow pitch (pitch $2 \mathrm{~F})$ is still required. In our approach, the MTJ stack is directly deposited on pre-patterned conducting pillars made of a nonmagnetic material for which the patterning process is already well mastered (e.g Ta pillars prepared by RIE or $\mathrm{Cu}$ or $\mathrm{W}$ vias prepared by damascene process). This way, the MTJ stack is then naturally patterned while being deposited thus not requiring any post-deposition etching. Some magnetic material is also deposited in the spaces between pillars. It can be left there without causing any problem. In this paper, we present the fabrication as well as structural, magnetic and electrical characterization (TMR, STT) of assemblies of such nanopatterned pMTJs. Despite this whole work was performed using upstream research clean room tools, the obtained results open a new path towards high density STT-MRAM.

\section{GROWTH OF MTJ ON PRE-PATTERNED POSTS}

\section{A. Fabrication of conducting non-magnetic posts}

As material for the pre-patterned non-magnetic posts, we chose Ta since its RIE is very well controlled. In order to avoid the risk of electrical shorts between pillars due to the material deposited in the trenches between posts, the latter are given an undercut shape (Fig.2). Thanks to this shape, during the MTJ deposition, no metal gets deposited on the pillar sidewalls nor at the foot of the metallic posts (see Fig.2b). The process for fabricating the conducting non-magnetic Ta posts with undercut is depicted in Fig. 3. Ta is coated by Pt to form the top part of the post and protect the top Ta surface from oxidation. Then, following the formation of cylindrical $\mathrm{Ta} / \mathrm{Pt}$ posts by an anisotropic RIE process, an isotropic RIE process is subsequently used in order to laterally trim the Ta part of the posts. By optimizing the experimental conditions (etching power, etching time, pressure, gas flow...), Ta posts of controlled dimensions with pronounced undercut were successfully fabricated (see Fig.3, 4, 7a). The depth of the undercut could be accurately controlled by the isotropic etching power (see Fig.4). This control allowed us to fabricate posts and patterned MTJs of diameter down to $\mathrm{F}=35 \mathrm{~nm}$ with pitch down to $1.5 \mathrm{~F}$ (Fig.5 and 8).. We also showed that low enough surface roughness can be obtained on top of the metallic posts by using $\mathrm{Pt}$ as etching mask rather than $\mathrm{Cr}$ mask (see AFM images in Fig. 6) allowing subsequent growth of the MTJ material.

\section{B. Growth of MTJ stack on the pre-patterned posts}

Following the fabrication of the $\mathrm{Ta} / \mathrm{Pt}$ posts, perpendicular MTJ stacks with $\mathrm{MgO}$ barrier were then deposited on these 
pre-patterned substrates (Fig.7). As expected, thanks to the undercut shape of the posts, no material is deposited at the foot of the posts providing good electrical insulation between neighboring MTJ cells. This novel nanopatterning approach therefore allows to form extremely dense arrays of electrically insulated MTJs with pitch down to $\mathrm{F}=1.5$ ( Fig. 8). This would be impossible to achieve with conventional IBE approach.

Another advantage of this approach is that one can benefit from the deposition of MTJ on pre-patterned posts to induce original properties. For instance, by depositing the $\mathrm{MgO}$ tunnel barrier at oblique incidence while rotating the substrate, it is possible to entirely coat the MTJ bottom electrode with $\mathrm{MgO}$ and get thicker $\mathrm{MgO}$ deposit on the sidewall of the bottom electrode than on the horizontal part of the MTJ (see Fig.9). This concentrates the current away from the edge of the nanopatterned MTJ thus reducing the influence of possible edge defects (see Fig.9). Similarly, lateral gradients of chemical composition can be created in the storage or reference layers. This can be used to induce different properties at the edges and center of each dot in order for instance to reduce nucleation effects at edges and improve STT switching efficiency.

\section{Structural characterization of MTJ stack on Ta posts}

The chemical composition and particularly the thickness uniformity of the tunnel barrier of these MTJ pillars were characterized by cross sectional transmission electron microscopy (TEM). $\mathrm{MgO}$ was here deposited at normal incidence. TEM images in Fig.10 show that the $\mathrm{MgO}$ tunnel barrier exhibits good continuity and thickness uniformity to the very edge of the pillar. They also show a slight contraction of the top electrode likely associated with the poor wettability of $\mathrm{CoFeB}$ on $\mathrm{MgO}$. This effect could be used to reduce the risks of electrical shorts across the tunnel barrier at the pillar edges.

\section{MAGNETO-TRANSPORT CHARACTERIZATION}

\section{A. Magnetic characterization}

The magnetic properties of pMTJ stacks deposited on pre-patterned substrates were evaluated by focused Kerr microscopy (Fig.11). Half-MTJ stacks were first deposited to characterize the interfacial perpendicular anisotropy of the deposits on top of the posts $\mathrm{CoFeB} / \mathrm{MgO}$ (see Fig. 11). By focusing the laser spot $(700 \mathrm{~nm}$ spot size) on a few pillars or on an area without pillars, the contribution of individual pillars and of the continuous MTJ material could be distinguished (Fig.11b and c). As usually observed, the coercive field of the patterned structures was larger than that of the continuous film of same composition. This difference in coercivity allows to unambiguously determine the switching field of the MTJ dots. This experiment revealed that the MTJs deposited on the prepatterned posts are out-of-plane magnetized as expected. For the same $\mathrm{CoFeB} / \mathrm{MgO}$ based MTJ composition, we then compared the coercive field distribution of an array of dots obtained by depositing the stack on a pre-patterned array of $\mathrm{Ta} / \mathrm{Pt}$ posts with the one obtained on an array of dots patterned y conventional IBE approach. As seen in Fig. 12, the switching ield distributions obtained by the two approaches are quite similar. However, the switching fields of the dots on the Ta/Pt posts are found overall to be slightly lower than those of the dots prepared by IBE. This can be ascribed to the bending of the $\mathrm{CoFeB} / \mathrm{MgO}$ layers at the pillar edges (see TEM images in Fig. 10) which may ease the nucleation of reversed domains when a reversed magnetic field is applied to the dots. Note that this does not necessarily implies reduced thermal stability.

\section{B. Transport characterization}

Next, the MTJs were electrically contacted (Fig.13) and electrical characterization of these patterned MTJs were performed in terms of TMR and STT switching characteristics. Typical magnetoresistance hysteresis loops of perpendicular MTJ stacks deposited on pre-patterned Ta/Pt posts without any post-deposition etching are shown in Fig. 14 versus magnetic field (Fig.14a) and voltage (Fig.14b). The field-voltage switching phase diagram at room temperature was measured and is shown in Fig.15. The color code represents the resistance state of the junction. The red color corresponds to stable antiparallel configuration (AP), the blue one to the stable parallel configuration $(\mathrm{P})$. The green is the bi-stable region where both $\mathrm{P}$ and AP configurations can be stabilized. At a constant applied field and even at zero field, the junctions can be switched from AP to P and from $\mathrm{P}$ to AP by STT. A switching voltage of $0.34 \mathrm{~V}$ for $100 \mathrm{~ns}$ pulse was measured which is quite comparable than in similar MTJs patterned by IBE [4]. These electrical results demonstrate that functional patterned perpendicular MTJs can be obtained by this novel patterning process consisting in depositing the MTJ material on pre-patterned conducting posts.

\section{CONCLUSION}

We have demonstrated a novel approach for nanopatterning MTJs down to sub-30nm dimensions at very narrow pitch by depositing the MTJ material on pre-patterned metallic posts. Despite this study was performed using upstream research clean room tools, TMR and STT switching of functional cells was demonstrated with characteristics similar to those of IBE etched MTJs. Remarkably, our approach allows to fabricate extremely dense arrays of very small size MTJs which is still impossible to achieve by IBE. Integrated in a CMOS process, the metallic vias obtained by damascene process which have naturally a V-shape could be used as the metallic posts on which the MTJ stack could be deposited. This opens a possible route toward high density STT-MRAM application. Besides, this approach can also be very valuable for other technologies of non-volatile memories such as PCRAM or RRAM by forcing the structural/chemical changes controlling the resistance variation to take place at well-controlled locations. This work was funded by the ERC Advanced Grant MAGICAL N669204.

\section{REFERENCES}

[1] M.Gajek et al, Appl.Phys.Lett.100, 132408 (2012)

[2] Y. Kim et al., "Integration of $28 \mathrm{~nm}$ MJT for $8 \sim 16 \mathrm{~Gb}$ level MRAM with full investigation of thermal stability," VLSI Symposium 2011, pp. 210-211.

[3]_M.Gaidis, "Magnetic back-end technology" in "Introduction to Magnetic Random Access Memories", IEEE Press Wiley (2017); ISBN 111900974X, 9781119009740

[4] A. A. Timopheev et al., Phys. Rev. B 92, 104430 (2015 


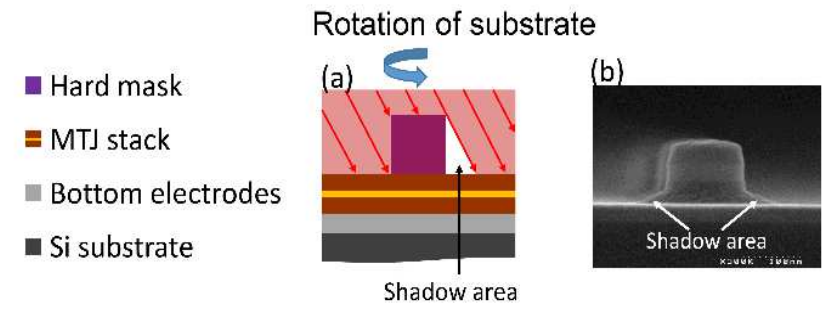

Fig. 1. (a) Conventional IBE process for the patterning of MTJ pillars. The material is etched at an angle to minimize redeposition on $\mathrm{MgO}$ sidewalls but this leads to shadowing effect yielding tapered sidewalls. (b) SEM image of a MTJ pillar patterned by IBE process.

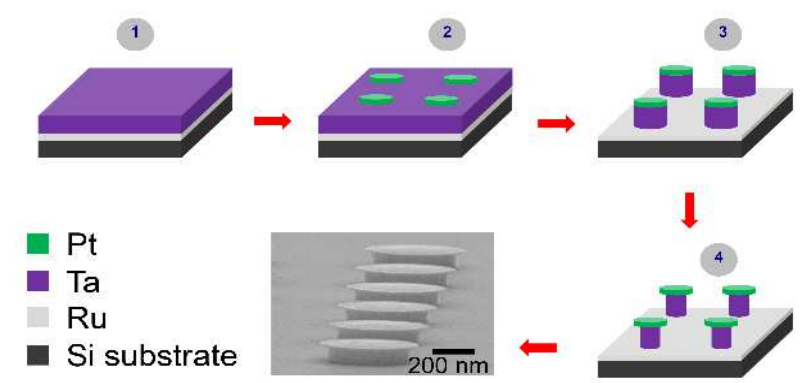

Fig. 3. Fabrication process of undercut Ta pillar using RIE. Typical SEM image of the undercut Ta pillars on Si wafer after RIE etching process. (a)

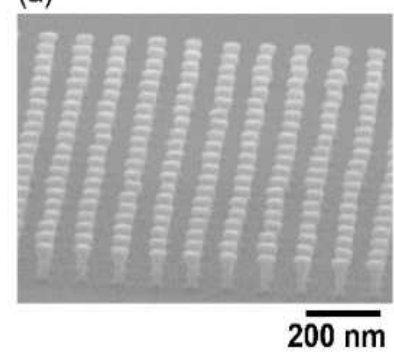

(b)

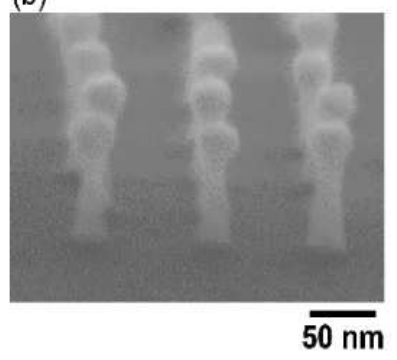

Fig.5. SEM images of very dense array of Ta posts with undercut: Diameter of $50 \mathrm{~nm}$ (a) and $35 \mathrm{~nm}$ (b). Fig. $5 \mathrm{~b}$ shows dense arrays of posts of size below $35 \mathrm{~nm}$ exhibiting a controlled $5 \mathrm{~nm}$ undercut (a)
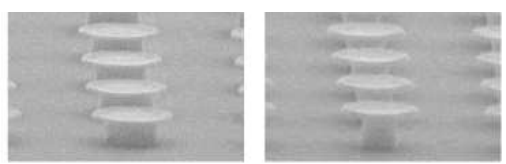

(b)

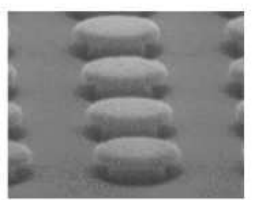

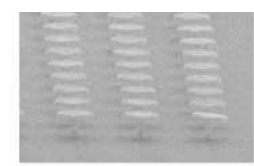

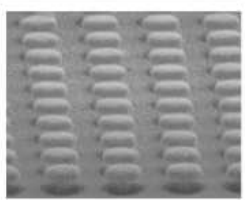

Fig. 7. Side view SEM images of arrays of pre-patterned Ta pillars of various diameters, (a) Before and (b) After MTJ stack deposition. One can clearly distinguish the material deposited on top of the pillars and in the spaces between them.

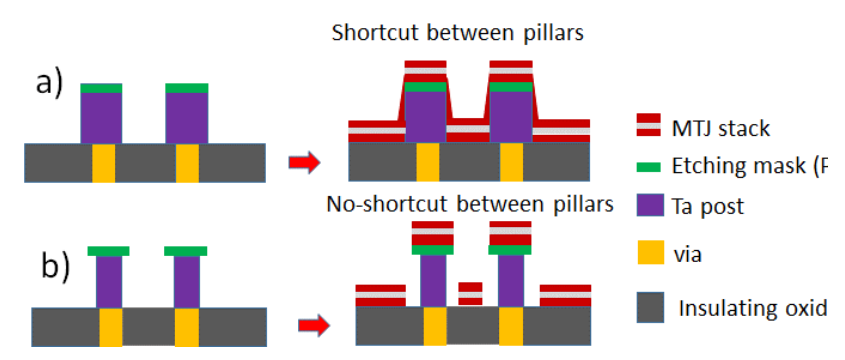

Fig.2: Illustration of the importance to pattern the non-magnetic conducting posts with undercut or V-shape. a) After depositing the MTJ material on posts without undercut, neighboring pillars are electrically interconnected due to metallic deposits at the foot of the posts. (b) When posts with undercut are used, no metallic material is deposited at the foot of the posts thus allowing good electrical separation between all pillars.

\section{Reducing isotropic etching power}
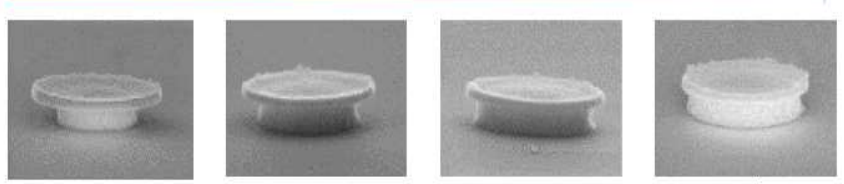

$20 \overline{0} \mathrm{~mm}$

Fig.4. Illustration of the control of the undercut by varying the isotropic etching power.

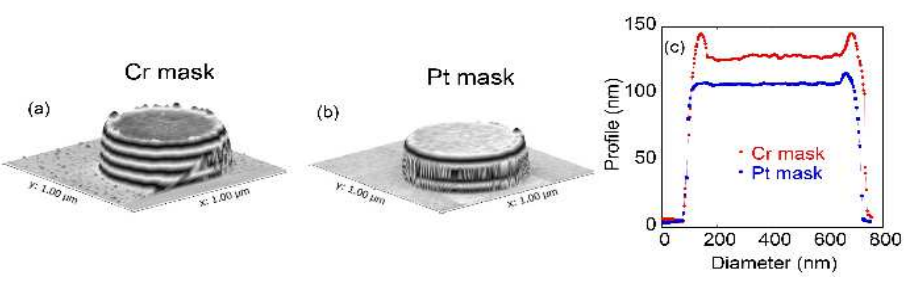

Fig. 6. AFM images and surface profile of Ta pillars for different etching masks $(\mathrm{Cr}, \mathrm{Pt})$. The RMS roughness with $\mathrm{Pt}$ is below $0.6 \mathrm{~nm}$. (a)

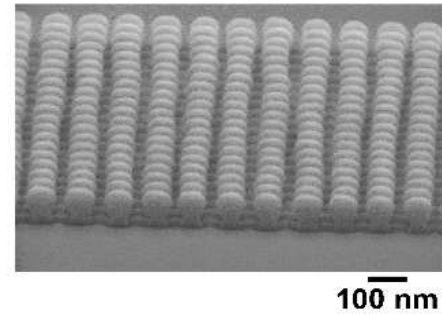

(b)

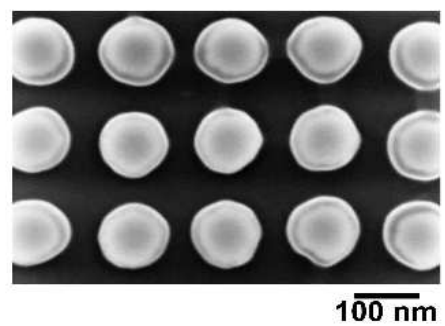

Fig. 8. SEM images of $\mathrm{F}=80 \mathrm{~nm}$ diameter of extremely dense MTJs arrays (pitch $=1.5 \mathrm{~F}$ ) deposited on pre-patterned $\mathrm{Ta} / \mathrm{Pt}$ posts: (a) Side view and (b) Top view. 


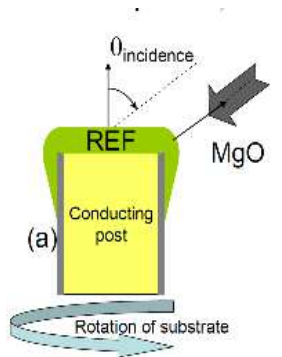

Thicker $\mathrm{MgO}$ deposit at edges than in the middle of the pillar since deposition rate varies as
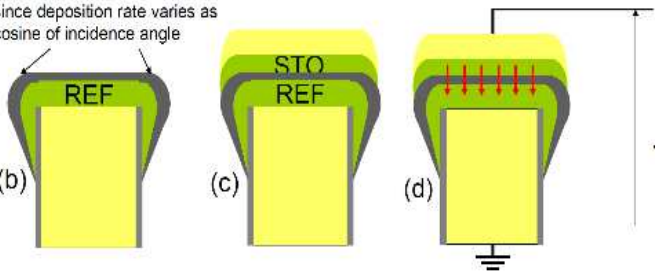

Fig. 9. (a-d): Deposition of the $\mathrm{MgO}$ barrier at oblique incidence to focus the current in the central part of the STT-MRAM cell. (a) After deposition of the bottom magnetic electrode by sputtering at normal incidence; (b) After deposition of the $\mathrm{MgO}$ layer at oblique incidence; (c) After deposition of the top electrode at normal incidence; (d) Representation of the current flow through the $\mathrm{MgO}$ barrier. The current flows mostly through the horizontal part of the $\mathrm{MgO}$ barrier which is the thinnest one. This can reduce the sensitivity of the electrical response to edge defects ad therefore the dot to dot variability

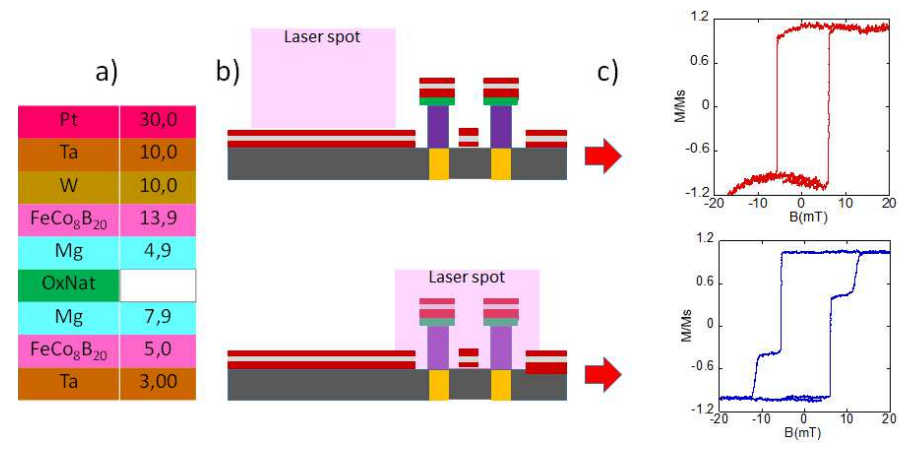

Fig.11. Focused Kerr measurements for detecting the magnetization switching of individual pillars. (a) Composition of the pMTJ $\mathrm{CoFeB} / \mathrm{MgO}$ stack deposited on the pre-patterned substrate (thickness in $\AA$ ). (b) Hysteresis loop of the continuous MTJ deposit (c) Hysteresis loop measured when the laser spot is focused on few pillars. Both contributions from the MTJs on top of the posts and from the deposit in the trenches between posts are visible.
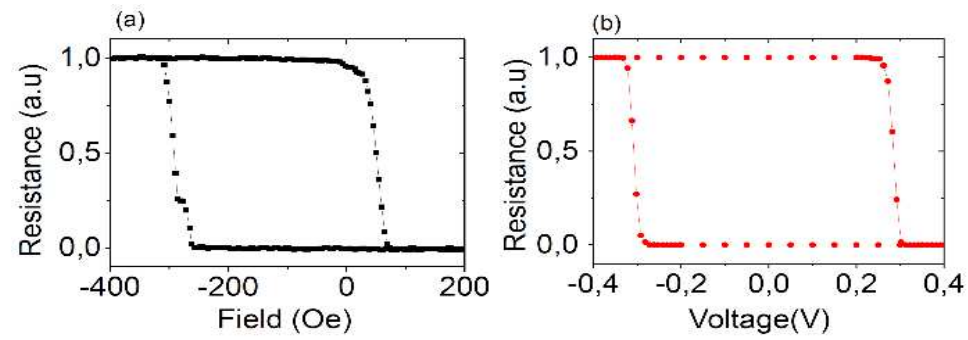

Fig.14. (a) Room temperature magnetoresistance loop of perpendicular MTJ stack deposited on pre-patterned Ta/Pt pillars versus (a) perpendicular magnetic field and (b) voltage.
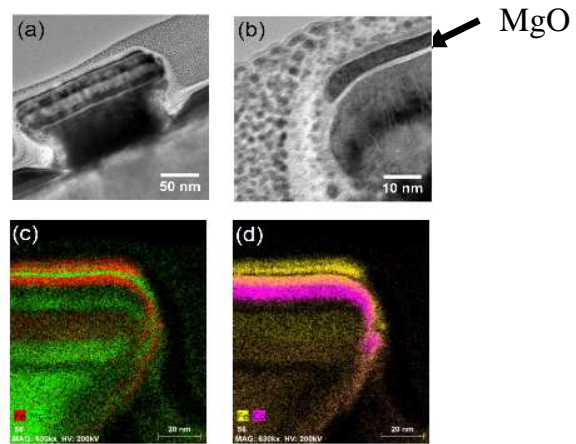

Fig.10. Cross sectional TEM (a, b) and chemical analysis (c,d) of the MTJ stack deposited on $200 \mathrm{~nm}$ pre-patterned Ta pillars.
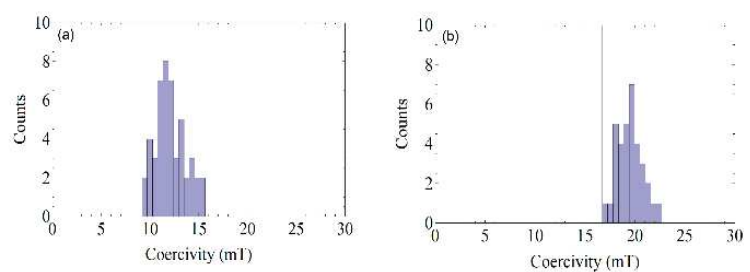

Fig. 12. Comparison of switching field distribution of (a) $\mathrm{CoFeB} / \mathrm{MgO}$ pillars grown on pre-patterned $500 \mathrm{~nm}$ Ta pillars and (b) Prepared by conventional IBE etching.
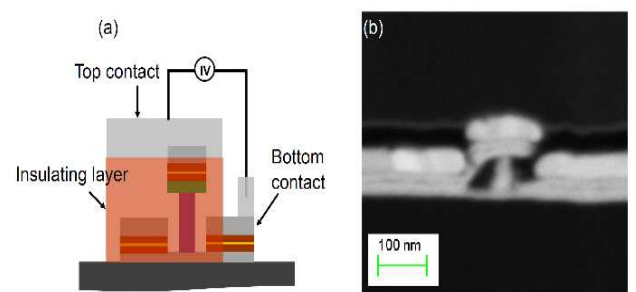

Fig. 13. (a) Illustration of MTJ stack on Ta post with electrical contacts for transport measurements. To electrically contact the MTJs, the trenches between the pillars were filled with a planarizing resist ACCUFLO. This step was followed by a resist etch-back process to open the top contact and by the patterning of the top contacting electrodes. The contacted devices were then electrically characterized (b) SEM cross section view of a device for electrical test.
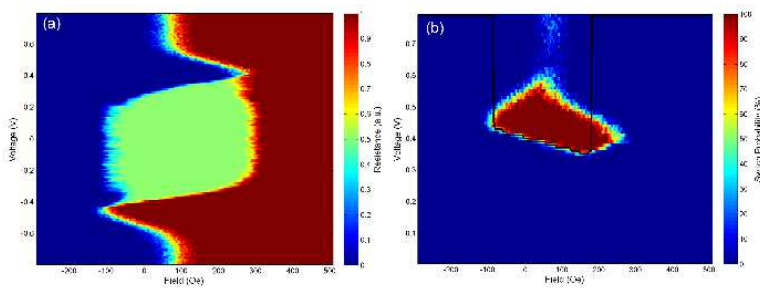

Fig. 15. (a) Typical field-voltage phase diagram of junctions deposited on pre-patterned $\mathrm{Ta} / \mathrm{Pt}$ posts, measured at room temperature. At each field point, a sequence of 100-ns writing pulses of increasing amplitude between $\mathrm{V}_{\min }=-0.8 \mathrm{~V}$ and $\mathrm{V}_{\max }=+0.8 \mathrm{~V}$ are applied to the junction. Between each pulse, the resistance is measured at low voltage. Once a voltage scan is completed, the field is incremented. To reduce the effect of stochasticity, the switching phase diagrams are averaged over 12 full measurements to obtain the final phase diagram (b) Probability of fieldvoltage switching window. 